\title{
Fluctuating Intelligence Bioinspired 3D Printed Design on Textile
}

Gabriele Pontillo ${ }^{1}$

Università degli Studi

della Campania Luigi Vanvitelli

gabriele.pontillo@unicampania.it

ORCID 0000-0001-5839-7838

\author{
Carla Langella ${ }^{1}$ \\ Università degli Studi \\ della Campania Luigi Vanvitelli \\ carla.langella@unicampania.it \\ ORCID 0000-0002-4346-656X
}

\begin{abstract}
Since its appearance in the world of design, 3D printing has been acclaimed as a new opportunity to free design thinking from the constraints imposed by traditional production processes. Over the past decade, additive systems have been applied in a variety of cultural and production contexts, crossing the boundaries of industry and beyond the semi-artisan dimension that has long characterized them. If $3 \mathrm{D}$ printing is now recognized as one of the production methods of the future, it is necessary to question the next prospects and especially the future of the relationship between design and additive technologies.

This paper intends to propose the scenario of the use of additive technologies of direct printing on fabrics as a new frontier of design and production that allows the development of changeable, flexible and composite artifacts increasingly related to the multi-functionality of nature and the human body and increasingly adaptable to the complexity of the needs of contemporary living.
\end{abstract}

\section{Keywords}

3D Printing on textile

3D pattern

Biomimetic

Medical design

Advanced Design 
Since its appearance in the world of design, 3D printing (3DP) has been acclaimed as a new opportunity to free design thinking from the constraints imposed by traditional production processes.

In the first years of their use, additive technologies proved to be a valid solution for the production of complex objects, unique or in small series, using plastic, ceramic and even metallic materials with the great advantage of not having to deal with problems traditionally related to the production of these materials such as undercuts, shrinkage, the need to produce molds, the difficulty of connecting different materials and elements. A realization emancipation that was immediately interpreted as expressive freedom and opportunity to experiment in different research and production contexts.

While 3DP was once mainly relegated to fablabs and makerspaces for prototyping, today the difficulties of using additive technologies in industrial production have been partly overcome and that semi-artisan dimension, which characterized the diffusion of these technologies in the early years, has greatly expanded.

As these technologies have become economically more accessible and technically more precise, varied and performing additive systems have progressively conquered new fields of application translating into tools for innovation in the production system (Jiménez et al., 2019). This expansion follows the indistinct and ubiquitous diffusion of the digital, that, together with Additive Manufacturing (AM), have assumed an important role in the innovation pathways of advanced manufacturing systems, while still maintaining that experimental character that helps find solutions to very specific problems, often in a faster, cheaper and more sustainable way, than conventional methods and technologies. Approaches such as automated and territorially distributed (Amed et al., 2017), bring about rapid changes in the traditional supply chain, favoring shorter time frames for the development of increasingly intimate interactions with consumers. This also becomes possible thanks to the hybridization between contexts and skills, generated by the cooperation between academic research environments, manufacturing companies, technologists, fablabs and makerspaces.

If $3 \mathrm{D}$ printing is now recognized as one of the production methods of the future, it is necessary to ask ourselves about the next prospects and especially about their repercussions in the relationship between design and additive technologies.

The paper intends to describe, through the account of the results of an experiment conducted within the Hybrid Design Lab ${ }^{2}$ of the University of Campania "Luigi Vanvitelli" entitled Fluctuating Intelligence, the opportunities and potentials of direct 3D printing on fabric using Fused Deposition Modeling (FDM) additive technology, in particular with machines of the low-cost category. In the course of the experimental research 3DP has proved to be a valuable opportunity to modulate optical characteristics, stiffness, deformability, self-cleanability, breathability of fabrics through direct printing of three-dimensional patterns. The experimentation was conducted using a biomimetic approach, that draws inspiration from biological structures referring to different types of three-di-
This article was discussed and agreed by the two authors and was written having shared the bibliography, readings, researchs and refl ctions. Carla Langella is responsible for the paragraph

"Bioinspiration and direct textile printing" and "3D printing on textile". Gabriele Pontillo is responsible for the paragraph "Application scenarios: Adaptable medical devices", "Processes and procedures" and "Technical stratagems". The introductory paragraph and the Conclusions, were jointly written by the two authors.

2

Lab founded in 2006 by Carla Langella at the Department of Architecture and Industrial Design, University of Campania "Luigi Vanvitelli", dedicated to the mutual relations between design and science with particular attention to the experimentation of biomimicry in design and the integration of designers in the development processes of new materials to which the specific esigner in Lab project is focused. 
mensional biological tissues and with the aim of proposing a wide range of solutions to meet the specific needs of some areas of application from lighting to biomedical.

\section{D Printing on Textile}

One of the most recent frontiers of experimentation related to $A M$ is the direct printing on textile materials whose potential and fields of application are still largely to be investigated. These techniques were born as a side effect of experimental activities on 3DP conducted in the field of fashion design by companies producing 3D printers in collaboration with artists and designers, as highlighted by the collaboration between Neri Oxman, Iris Van Herpen and Stratasys in 2013, which outcome was Anthozoa, a collection enriched by $3 \mathrm{D}$ bioinspired elements, generated using an algorithmic approach to emphasize the relative movements of the different parts of the body. In line with this experience, the European Project Re-FREAM was developed, funded by the European Community in the Horizon 2020 program in 2019, proposed by the universities of Berlin, Linz and Valencia involving artists, scientists and designers, oriented to innovate the textile supply chain through additive manufacturing technologies.

In addition to these experiments of mainly formal nature on the topic of 3DP on fabric, several more technological researches are underway aimed at testing the physical/mechanical properties of the artifacts made with these new processes in order to provide operational protocols to be used with different types of technologies and materials (Spahiu et al., 2017; Schmelzeisen et al., 2018; Ayvali et al., 2020; Loh et al., 2021).

From the state of the art of 3DP on textiles it emerges, therefore, that interest in this type of technique is growing, but that the potential so far tested is still only a fraction of what could be achieved. In terms of areas of application, however, direct 3DP on fabric has been used mainly in fashion design with the aim of creating complex and dramatic geometries, released from the two-dimensionality of traditional fabrics, easily customizable according to the needs of wearability and morphological and biometric characteristics of the human body (Zolfagharifard, 2013). However, the potential of these techniques goes far beyond the world of fashion and its spectacularity, because they hint at new and unprecedented opportunities for the advancement of the entire design and production scenario of additive technologies, as they would allow to reduce production time compared to artifacts made exclusively in 3DP, and to improve technical-functional performance (Grimmelsmann et al., 2016).

3DP on fabric can be applied to different types of natural and artificial fabrics. The selection of the fabric involves an in-depth study of the interaction between the physical, chemical or mechanical properties of the fabric (e.g. elasticity, thickness, porosity, surface microstructure) and those of the 3D printed component. Design based on a conscious functional combination of these variables offers the opportunity to obtain complex and unusual performance, difficult to develop with traditional processes (Chatterjee \& Ghosh, 2019). 
In addition to the selection of the textile substrate and the design and modeling of the pattern to be printed, there is the choice of the printing technology and the material, or materials, to be used. Although current 3DP technologies use a variety of polymeric materials, not all of them are suitable for textile applications, since the type of compatible textile substrate varies as the extrusion and deposition temperatures change. Some synthetic textile materials in particular, risk being damaged if placed in contact with the first layer of extruded material with a minimum temperature of 160 degrees. From the experiments carried out so far, it emerges that the most versatile and suitable technologies for this type of printing are Polyjet and Fused Deposition Modeling (FDM) since, depending on the polymer chosen, it is possible to reduce the melting and extrusion temperature, especially when materials such as Polylactic Acid (PLA) and Polycaprolactone (PCL) are printed.

\section{Bioinspiration and Direct Textile Printing}

The direct-on-fabric printing scenario is largely yet to be invented. If the input of 3DP led us to think that it was the paradigm that, as never before, would allow us to imitate nature, today with direct 3DP on fabric this is even more true because through it, design conquers the possibility of adopting biological textiles with mixed rigid-flexible components such as the skins of some animals on which scales are grafted, scales (flakes), claws or fur of different materials. Compared to the past, technology is proposing instruments of investigation with increasing resolution that go as far as to observe nature down to the nanoscale, revealing its most intimate details. From these details, scientists are able to deduce new knowledge about the processes and principles that underlie the beauty of biological structures. The beauty of nature is related to its intelligence and is a composite, multifunctional beauty at every dimensional scale.

In the biological tissues present in nature, in both animate and inanimate organisms, a wide variety of colors, patterns, ornamentation, layering, roughness, cilia layers, porosity, scales, geometries, and shapes are present. Recent scientific studies have revealed that these characters, apparently aesthetic and formal, respond to principles of functionality and efficiency as well as communicative intentions oriented to biological objectives such as sexual attraction or concealment from predators. Through increasingly precise spatio-temporal models, scientists are uncovering the physical-chemical (transport, chemical gardens, periodic precipitations and Turing model), mechanical-structural and morpho-generative principles and logics underlying the formation of nature's twoand three-dimensional patterns (Serna \& Barragán, 2017).

A particularly interesting reference for the field of 3D printing on textiles are the armor shells of some animals that mainly perform the function of protecting the organism mechanically from the surrounding environment, including attacks from potential predators. In addition to this protective function, however, there are other functions such as hydrodynamic drag reduction, coloration, and tactile and optical sensory perception. Often these armors in order 
to ensure the coexistence of protection, articulability and other functions, are composed of a multitude of scales more or less rigid, with highly functionalized morphologies that can be overlapping as in pangolins or in some snakes and fish (Domel, 2018) or abutting as in osteoderm animals like turtles, lizards, alligators, and armadillos (Connors et al., 2019).

In order to identify and apply morphological-functional analogies between these natural structures and artifacts, the project must be based on a detailed and deep knowledge, at different dimensional scales, of the morphological, material and mechanical characteristics of biological tissues. An in-depth knowledge and a critical reading of the functional effects that requires the intervention of biologists and possibly also of engineers who support the designers by performing experimental investigations through the use of the wide range of experimental and modeling approaches currently available, such as electron microscopy, X-ray micro tomography, nanoindentation, mechanical testing, and finite element modeling.

Such approach goes far beyond the imitation of biological systems and requires the discovery of the fundamental principles that govern the hidden efficiency of nature.

Some examples of properties that can be obtained by integrating the biomimicry approach with 3DP on textiles are: self-cleanability inspired by the lotus effect of leaves; resistance to surface wear inspired by the skin of crawling animals; tensile strength inspired by the auxetic skins of some macrophage reptiles; heat dissipation inspired by leaves or iridescence inspired by the structural color of some insects.

\section{Application Scenarios: Adaptable Medical Devices}

Born in the world of fashion, the techniques of 3DP on fabric are particularly suited to be used in the design sectors where artifacts must interface with the body and its specificities such as sport protections ${ }^{3}$ and medical aids.

It is for this reason that the Fluctuating Intelligence project is focused on these two fields, with the intention of designing new high-performance wearable devices suitable for different contexts and tailored to specific needs, using direct-to-fabric 3DP via FDM additive technology.

In particular, for the field of biomedical devices, the analysis of the scientific literature and the relevant state of the art (Pei et al., 2015), as well as direct discussions with the team ${ }^{4}$ of orthopedic physicians in the Multidisciplinary Department of Medical-Surgical and Dental Specialties with whom HDL collaborates on an established basis, revealed that many of the arthritic and inflammatory conditions are treated with orthotic devices that the patient can wear independently. Such devices often present problems of compliance, acceptability, and comfort. The possibility of grafting elements or patterns of rigid or semi-rigid elements on elastic, flexible and breathable fabrics allows to conceive a new generation of lightweight aids, adaptable to the morphology of the body, customizable and comfortable and also integrated with clothing and accessories.

\section{3}

In sports where impacts are expected, such as skating, skateboarding, skiing, horseback riding, use of protections that tend to be uncomfortable, bulky, aesthetically unpleasant, are required and therefore not used willingly. 3D printing on fabric allows the protection to be incorporated directly on sports clothing or accessories so as to be more easily wearable, integrated with the look and style of the specifi sport, made to measure and configu ed according to needs.

4

Coordinated by Prof. Giovanni Iolascon. 
As part of the experimentation conducted, this approach was used to create a wrist brace and a knee brace.

The wirst brace Fig. 1 was developed for the therapeutic immobilization in case of traumatic, inflammatory and degenerative diseases of the hand and wrist such as rhizoarthrosis and De Quervain's syndrome. Generally, these aids consist of a bandage of elastic fabric that wraps around the wrist, to which rigid elements can be added that accompany or support the wrist inducing it to maintain a correct position, but the experimentation allowed to highlight the possibilities offered by the system of direct 3DP on fabric to graft the rigid, semi-rigid or flexible elements directly on the textile in a very precise and personalized way on the individual patient, managing morphologies and dimensions of these grafts according to the specific therapeutic indications required by the rehabilitation of the inflamed or compromised joint.

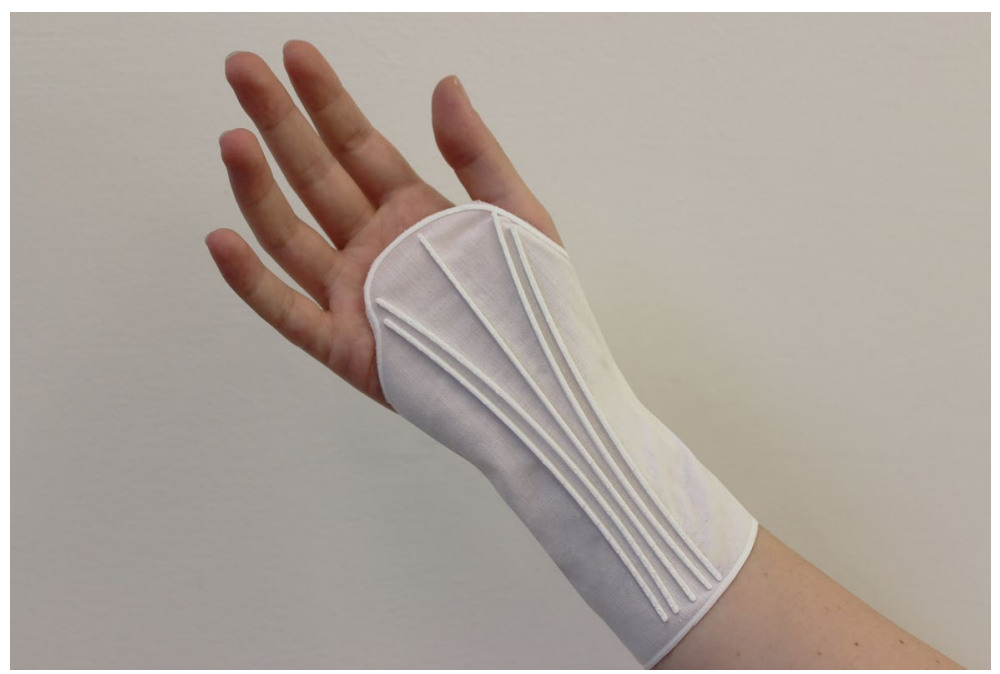

Fig. 1

Gabriele Pontillo,

Wrist brace.

Design of a custom wrist orthosis consisting of lamellar bioinspired geometries printed directly on fabric. The design, bioinspired by the structure of waterbirds webbed feed, guarantees through the linear elements to keep the wrist rigid and at the same time to bandage it with a light and $\mathrm{fl}$ xible material, such as cotton used in this case as a substrate.

(C) Gabriele Pontillo, Carla Langella.

Once the first device had been developed, a design protocol was outlined, starting with a deep transdisciplinary survey of the anatomical part to be treated (based on 3D diagnostic images and therapeutic indications), and then developing by 3D modeling software the structure of the aid based on adding to the planar fabric an array of rigid longitudinal elements (ribs) to modulate support and mobility. The thickness and morphology of these ribs derive from the indications of physiatrists on the movements and micro-movements that are allowed or impeded. The result obtained from the design process described and the subsequent printing on elastic fabric, allows to obtain a perfect integration and mechanical collaboration between the substrate - flexible part - and the ribs - rigid part. The use of the direct-to-fabric 3DP process in this context shows a significant advancement compared to the state of the art, as it would allow to obtain, in less time and with significantly lower costs, performing, lightweight and breathable orthotic devices. 
Regarding the economic aspect, it is important to underline how the reduction of time and costs is due to the modeling and printing of the artifact in a planar mode which acquires three-dimensionality when worn. In this way, the complexity of the anatomical part has a reduced impact compared to custom $3 \mathrm{D}$ printed braces that require longer time, supports, and obviously much more material.

The design of the knee brace Fig. 2 was developed using the same approach with the goal of providing a lightweight and comfortable aid to people with inflammation or microinjuries of the knee ligaments who still intend to conduct sports activities, albeit with caution. The 3D printed ribs on the textile material prevent complete knee eversion or relative lateral movements that could worsen the condition of the ligaments. The design was inspired by knee muscles and tendons structure and by the girdle of the chiton Rhyssoplax canariensis Fig. 3.

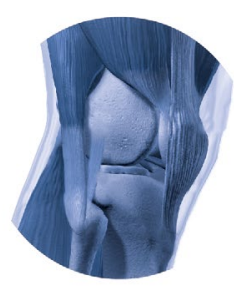

3D

Survey of the anatomical part to be treated

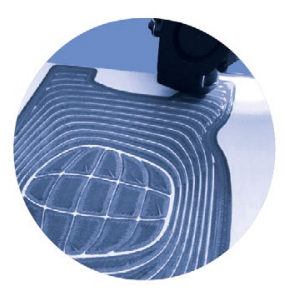

2D

Direct-to-fabric 3DP process

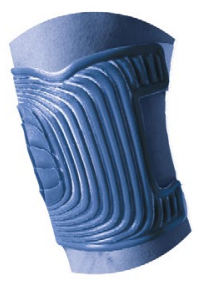

3D

Knee brace printed and worn

\section{Knee Brace}

bioinspired design approach

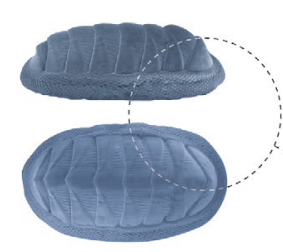

ANATOMICAL MODEL ANALYSED Poliplacofori, noliplacofori, Chitons

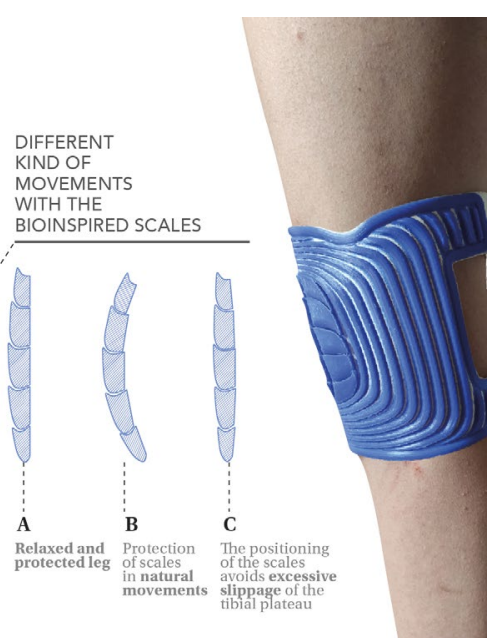

Fig. 2

Salvatore Carleo, Knee Brace - innovative design and production technique.

The diagram highlights the design process that starts with an anatomical survey of the patient's knee. From the 3D survey of the knee, the brace is modelled in 2D, printed in a planar manner using the direct-to-fabric 3DP technique, and then closed via the external flaps is orn by the patient.

Acknowledgments:

Degree Thesis in Design for Innovation carried out at the Department of Architecture and Industrial Design (DADI) of the University of Campania "Luigi Vanvitelli".

Supervisors Prof. Patrizia Ranzo and Carla Langella. Referee Gabriele Pontillo (Design and production technique).

Fig. 3

Salvatore Carleo, Knee Brace.

Scheme of a bioinspired approach that strarts from the analysis of the central structure of the Chiton R. Canariensis (Scientific imag reference: Connors et al., 2019). The protective function of the central part of this organism was transferred to the design of the knee brace, which is designed to support the anterior cruciate ligament $(A C L)$ and protect it during sports activities. The peculiarity of the developed geometry is the possibility to bend the knee within a certain limit, guaranteeing normal mobility, while blocking all those movements that are harmful to the ACL. Acknowledgments: view Fig. 2. 
The purpose of this research was therefore to study, on the one hand, the possible applications and areas in which this technique can be used through the analysis of specific structural and functional logic to which the object/fabric must respond, and on the other hand to study issues more closely related to technology such as adhesion of the polymeric material printed directly on the fabric using a low-cost FDM machine Fig. 4. In order to do this, some samples have been designed and prototyped, useful to analyze
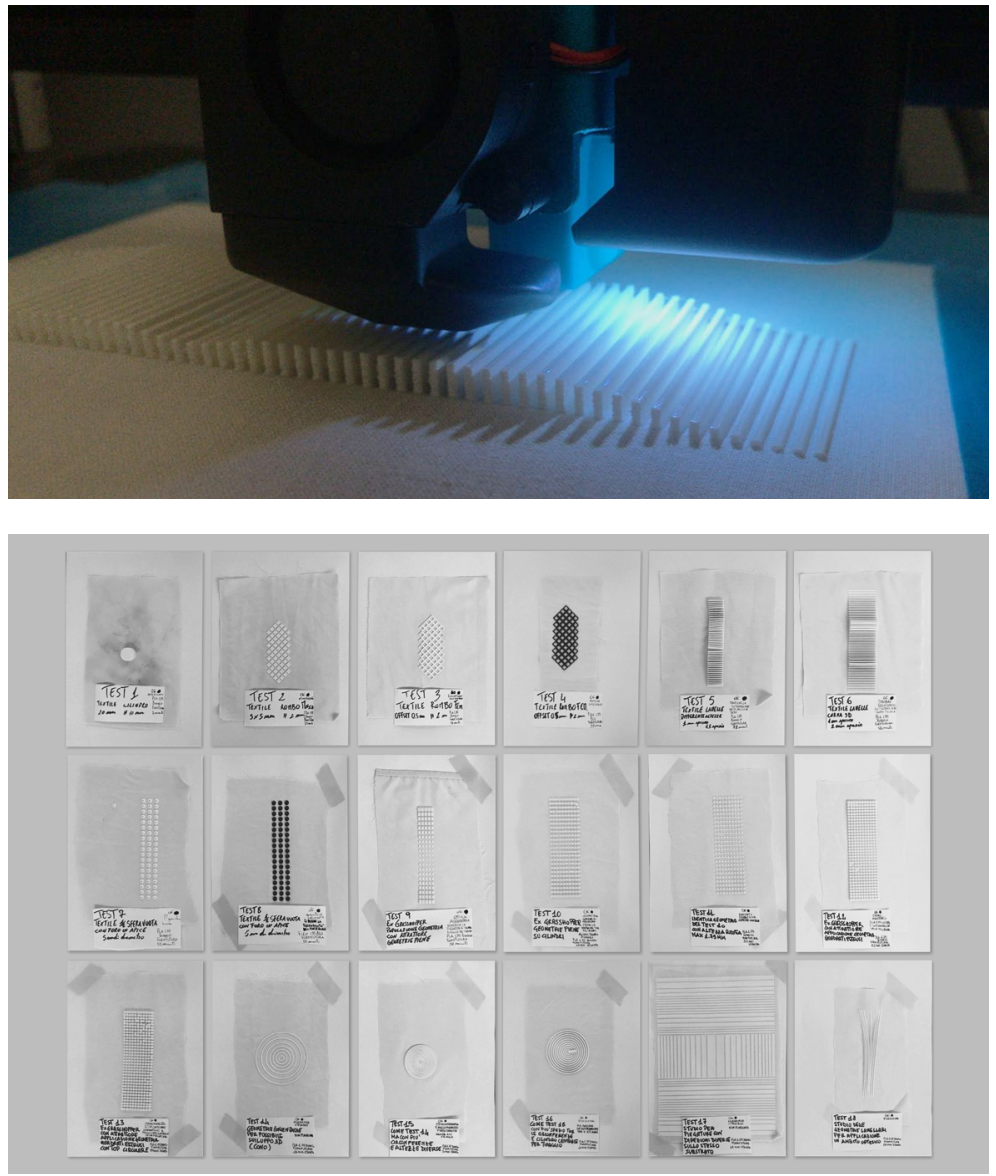

the limitations of this technique and identify potential applications Fig. 5. Among these, medical braces are also included, in which the flexibility and breathability of the fabric, together with the stiffness of the structures consisting of extruded polymer, provide comfort and support to the designed device, an area that is believed to open up many avenues for customization and design of orthosis, according to specific needs.

The outlined fields of intervention and their possible applications, require a thorough understanding of the processes and procedures to be used in this type of technique, which not only
Fig. 4

Gabriele Pontillo, Direct 3D Printing on Textile.

The image shows the innovative process of direct-to-fabric 3DP using low-cost FDM additive technology.

(C) Gabriele Pontillo, Carla Langella.

Fig. 5

Gabriele Pontillo, Sample test.

The tests performed are instrumental in understanding the behaviour in terms of form and adhesion of the 3D printed part, in PLA or TPU, on the fabric substrate. In particular, it is shown how, depending on the type of shape of the geometry, aligned or not with the warp and weft of the fabric, it is possible to obtain di erent behaviours that can increase, rather than invalidate, the textile qualities of the substrate and therefore the functionality of the project: concentric circles, for example, sti en the fabric, small juxtaposed elements or lamellae, maintain its fl xibility.

(C) Gabriele Pontillo, Carla Langella. 
requires specific modeling skills and development of 3D geometry, but also a deep knowledge of software and instrumentation. The state of the art and the scientific literature of reference, highlight how, at first, the experiments conducted, involved the use of stereolithography (SLA) for direct printing on fabric (Grothe et al., 2020). Recently, the opening to FDM printers and in particular low-cost ones is providing a strong boost to the diffusion of this technique making it more accessible thanks to significantly reduced costs for the companies producing aids.

Direct 3D printing on fabric, thanks to the use of FDM printers, can therefore be easily scalable and extended to different areas of application and types of artifacts, precisely because it allows, through three-dimensional geometric and parametric modeling, the management of complex and differentiated structures and patterns according to specific needs Fig. 6.
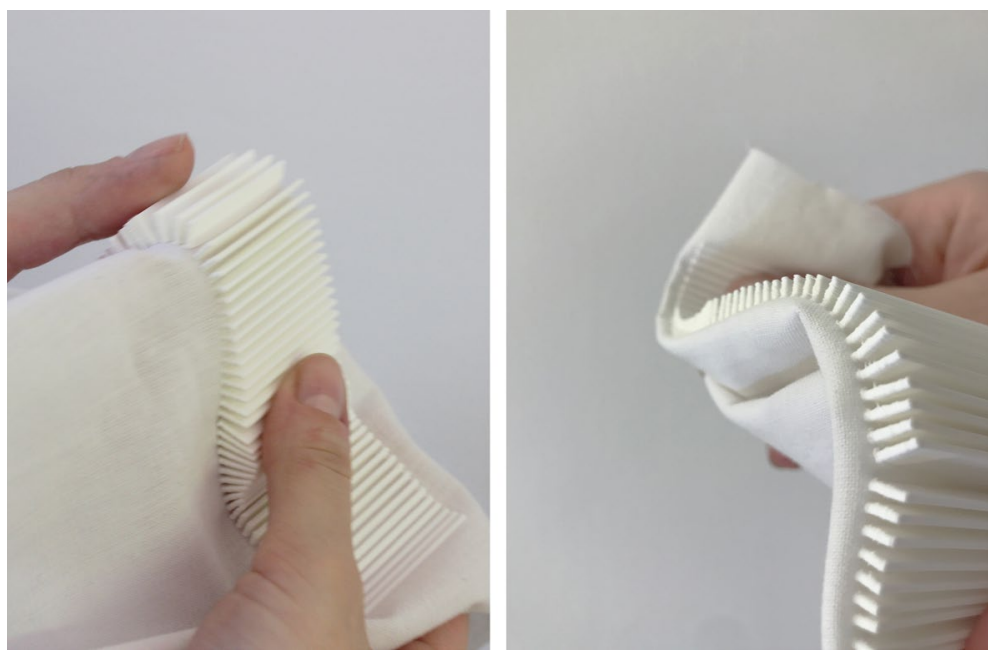

At this point, it is important to examine the parameters and technical design and manufacturing choices useful to optimize this process. Not all materials are suitable for direct-on-textile 3DP; similarly, not all textile materials can provide good adhesion of the part being 3D printed (Malengier et al., 2018).

Preliminary tests performed during the experiment were useful to understand the adhesion properties of 3D printed patterns with different morphologies, configurations, and sizes on different textile substrates. The materials chosen for the printed tests were PLA and Thermoplastic Polyurethane (TPU), while the textile substrate used was $100 \%$ cotton, with a thickness of $0,48 \mathrm{~mm}$.

This gave the possibility to print with a standard $0,40 \mathrm{~mm}$ nozzle. For the tests, a low-cost Cartesian FDM printer was used, with a thread puller incorporated in the extruder body, so as to allow to print TPU as well as PLA. Tests were also conducted on less smooth substrates, with surface hair, such as wool, which proved to be unsuitable for FDM 3DP, since the protruding fibers, flattened by the passage of the extruder, tend to return later to their original position due to elasticity. This shape memory of the fabric leads
Fig. 6

Gabriele Pontillo,

Flexibility test.

Flexibility analysis of geometries printed directly on fabric.

The test, consisting of variable-height flap generated by a parametric process, highlights the fl xing possibilities of the fabric despite direct printing on the substrate. Great potential for development and application can be achieved by a thorough knowledge of the printed material and the substrate being used. (C) Gabriele Pontillo, Carla Langella. 
the printing material, still hot and flexible after deposition, to deform, causing a poor adhesion of the latter on the fabric substrate and affecting the final yield of the printed part which tends to come off. Other tests have been conducted on cotton substrates coated with acrylate, on which the PLA did not adhere. These experiments highlight the issue of the interface between the printed material and the support material that is generally not considered in canonical 3DP where instead detaching agents are used to prevent the printed objects from sticking to the plate.

\section{Technical Stratagems}

The problem of the interface between fabric and printed material is not only related to the type of material but also to a dimensional issue that must be addressed with particular attention. In order to not cause an excessive scraping on the textile substrate of the nozzle, whose force of movement and traction risks causing the displacement from the printing plane, a $100 \%$ cotton with a thickness of $0,48 \mathrm{~mm}$ was chosen. This allowed to set the layer height to 0,40 $\mathrm{mm}$ avoiding the entanglement of the fabric. A greater distance between substrate and nozzle, instead, would not have guaranteed the good rooting of the plastic to the fabric. As for the methods of fixing the fabric to the printing bed, different ones were tried such as lever clamps, glue, double-sided tape and high adhesion scotch paper. From the tests carried out, high adhesion scotch paper was chosen, as it gave the best results, both for fixing the substrate and for removing it from the printing bed.

PLA is usually printed with a temperature ranging from $190^{\circ} \mathrm{C}$ to $205^{\circ} \mathrm{C}$, a parameter that varies depending on the characteristics of the filament used, but it has been noticed that, setting the extrusion temperature about $5^{\circ} / 10^{\circ} \mathrm{C}$ higher than the standard of the chosen material, allows a better infiltration of the extruded plastic into the weave of the cotton substrate.

Among the first parameters that were changed in order to print on the textile substrate, is the flow rate of the PLA material, which was reduced so to provide the ability for the molten plastic to penetrate the fabric and consequently fill the entire weave of the substrate. So also the printing speed of the first layer on the $X / Y$ axes was reduced in order to give the PLA material more time to be able to take root on the substrate. These two values are also directly proportional when printing without a substrate. By decreasing the speed of the first layer and the extrusion flow of the material, the cooling fan of the material was deactivated in order to avoid the risk that the rapid cooling of the printed material could affect the adherence of the printed part to the substrate, and cause a deformation due to the retractive force of the extruded polymeric part. 


\section{Conclusions}

Additive printing technologies have been recognized by the design world as the most suitable for mimicking biological morphologies and structures. 3DP on textile substrate, in particular, allows the creation of surfaces with macro characteristics similar to biological ones with adaptability, complexity and multidimensionality characteristics that are difficult to achieve with traditional textile technologies. Nature, therefore, is the ideal reference for this type of process because it offers a huge repertoire of references of combinations between flexible fabrics and rigid or semi-rigid superstructures.

Despite what may be the limitations of the FDM technology, particularly in terms of resolution and printing quality, the possibility of freely experimenting with different materials and substrates makes the choice of low-cost additive technologies suitable both for research activities and for those connected with testing and greater dissemination of the products made ${ }^{5}$.

Through the transfer of three-dimensional patterns borrowed at the micro and macro scales from nature's surface structures, such as biological skins and fabrics, it is possible to obtain materials that are similar not only in appearance, but also in functionality and versatility, as well as homogeneous patterns with homogeneous functionalities or patterns differentiated by structural and functional gradients that allow to modulate on the textile surface technical characteristics such as deformability, adherence, breathability, wear resistance, hydrophobia or self-cleanability. All these characteristics can be obtained by configuring the combination of biomimetic reference, geometry of the pattern to be printed, selection of the support fabric, definition of technologies and process parameters. The modulation of these parameters becomes the subject of a new complex advanced design that integrates digital skills, material but also human based, adequate to respond to the complexity of products that interface with the body such as medical products that require particular attention to the properties of adaptability, comfort, and acceptability.
In this regard, it should be noted that what is described in this contribution is part of a project that is still in progress, and which is currently focusing on the use of alternative substrates, such as a composite based on Polyester and Polyurethane.

\section{Gabriele Pontillo}

Italian product designer. In 2019 he obtained a Doctoral Research Fellowship in Environment, Design and Innovation at the Università degli Studi della Campania "Luigi Vanvitelli". Main focus of his line of research are parametric design, medical design, and advanced manufacturing - knowledge acquired during his academic path.

\section{Carla Langella}

Architect, Ph.D., Associate Professor of Industrial Design at the Department of Architecture and Industrial Design, Università degli Studi della Campania "Luigi Vanvitelli", where she teaches Bio-innovation Design and Design for Scientific Visualization in the Master's Degree Course Design for Innovation and Industrial Design Laboratory 3. 


\section{References}

Amed, I., Berg, A., Brantberg, L., Hendich, S., Leon, J., \& Young, R. (2017). The state of fashion 2017. McKinsey \& Company.

Ayvali, M., Bussieweke, L., Drunzinin, G., Korkmaz, M. \& Ehrmann, A. (2020). 3D printing on wrap-knitted fabrics. In ICTTE 2020 - IOP Conf Series: Materials Science and Engineering, 1031(2021), 012019.

Chatterjee, K., \& Ghosh, T.K. (2019). 3D Printing of Textiles: Potential Roadmap to Printing with Fibers. Adv. Mater. 2019, 1902086.

Connors, M., Yang, T., Hosny, A., Deng, Z., Yazdandoost, F., Massaadi, H., ... \& Li, L. (2019). Bioinspired design of flexible armor based on chiton scales. Nature communications, 10(1), (pp. 1-13).
Domel, A.G., Saadat, M., Weaver, J.C., Haj-Hariri, H. Bertoldi, K., \& Lauder, G.V. (2018). Shark skin-inspired designs that improve aerodynamic performance. Journal of the Royal Society Interface, 15(139), 20170828.

Grimmelsmann, N., Meissner, H., \& Ehrmann, A. (2016). 3D printed auzetic forms on knitted fabrics for adjustable permeability and mechanical properties. IOP Conf. Series: Materials Science and Engineering, 137, 012011. https:// doi.org/10.1088/1757899X/137/1/012011

Grotte, T., Brockhagen, B., \& Storch, J.L. (2020). Three-dimensional printing resin on different textile substrates using stereolithography: $A$ proof of concept. Journal of Engineered Fibers and Fabrics, 15, 1-17. https:// doi.org/10.1177/15589250 20933440
Jiménez, M., Romero, L. Domìnguez, I.A., del Mar Espinosa, M., \& Domìnguez, M. (2019). Additive Manufacturing Technologies: An Overview about 3D Printing Methods and Future Prospects. Hindawi Complexity, Volume 2019.

Loh, G.H., Sotayo, A., \& Pei, E. (2021). Development and testing of material extrusion additive manufactured polymer-textile composites. Fash Text, 8(2).

Malengier, B., Hertleer, C., Cardon, \& L., Van Langenhove, L. (2018). 3D Printing on textiles: Testing of Adhesion. Journal of Fashion Technology and Textile Engineering, Eng S4:013.

Pei, E., Shen, J., \& Watling, J. (2015). Direct 3D printing of polymers onto textiles: experimental studies and applications. Rapid Prototyping Journal, 21(5), 556-571.
Schmelzeisen, D., Koch, H., Pastore, C., \& Gries, T. (2018). 4D Textiles: Hybrid Textile Structures that Can Change Structural Form with Time by 3D Printing. In Y. Hyosev et al. (Eds.), Narrow and Smart Textiles. Springer International Publishing AG.

Serna, H., \& Barragán, D. (2017). Patterns in nature: more than an inspiring design. Revista de la Academia Colombiana de Ciencias Exactas, Físicas y Naturales, 41(160), 349-360.

Spahiu, T., Grimmelsmann, N., Ehrmann, A., Pieper, W., \& Shell, E. (2017). Effect of 3D printing on textile fabric. 1sr International Conference "Engineering and Entrepreneurship" Proceedings, ICEE-2017.

Zolfagharifard, E. (2013). When science is stylish: Designer uses 3D printing to create a range of accessories that is taking the fashion world by storm. Daily Mail. 